

\title{
Basic Aspects of High-Tc Grain Boundary Devices
}

J. Mannhart, H. Hilgenkamp, B. Mayer, Ch. Gerber, J. Kirtley, K. Moler, M.

Sigrist

\section{To cite this version:}

J. Mannhart, H. Hilgenkamp, B. Mayer, Ch. Gerber, J. Kirtley, et al.. Basic Aspects of High-Tc Grain Boundary Devices. Journal de Physique IV Proceedings, 1996, 06 (C3), pp.C3-329-C3-334. 10.1051/jp4:1996350 . jpa-00254268

\section{HAL Id: jpa-00254268 https://hal.science/jpa-00254268}

Submitted on 1 Jan 1996

HAL is a multi-disciplinary open access archive for the deposit and dissemination of scientific research documents, whether they are published or not. The documents may come from teaching and research institutions in France or abroad, or from public or private research centers.
L'archive ouverte pluridisciplinaire HAL, est destinée au dépôt et à la diffusion de documents scientifiques de niveau recherche, publiés ou non, émanant des établissements d'enseignement et de recherche français ou étrangers, des laboratoires publics ou privés. 


\title{
Basic Aspects of High- $T_{\mathrm{c}}$ Grain Boundary Devices
}

\author{
J. Mannhart, H. Hilgenkamp, B. Mayer, Ch. Gerber, J.R. Kirtley*, K.A. Moler*(1) and M. Sigrist** \\ IBM Research Division, Zürich Research Laboratory, 8803 Rüschlikon, Switzerland \\ * IBM Research Division, T.J. Watson Research Center, P.O. Box 218, Yorktown Heights, NY 10598, \\ U.S.A. \\ ** Theoretische Physik, ETH-Hönggerberg, 8093 Zürich, Switzerland
}

\begin{abstract}
Grain boundaries are extensively used as high-quality Josephson junctions in high- $T_{\mathrm{c}}$ superconductors. Their superconducting characteristics can generally be well described by conventional models of strongly coupled Josephson junctions. Here, we report on highly anomalous critical current vs. magnetic field dependencies of grain boundaries in $\mathrm{YBa}_{2} \mathrm{Cu}_{3} \mathrm{O}_{7-x}$. Direct imaging with scanning SQUID microscopy provides evidence of magnetic flux generated by single grain boundaries. Conventional Josephson junction models cannot explain these effects if a superconducting order parameter with a pure $s$-wave symmetry is assumed. The results have significant implications for our understanding of the properties of grain boundaries in high- $T_{\mathrm{c}}$ superconductors and for their applications.
\end{abstract}

\section{INTRODUCTION}

Single grain boundaries [1] are widely used as high-quality Josephson junctions in a variety of device applications and for experiments to investigate fundamental properties of the high- $T_{\mathrm{c}}$ cuprates, such as the symmetry of their superconducting order parameter [2-4]. In contrast to the textbook behavior of the commonly used $24^{\circ}$ grain boundary, characteristic features of large-angle grain boundaries are highly anomalous and cannot be explained by conventional Josephson junction properties and by a superconducting order parameter with $s$-wave symmetry. An example of these unusual properties is given by anomalous dependences of the critical current $I_{c}$ on an applied magnetic field $H_{\mathrm{a}}$. These peculiar characteristics can be accounted for remarkably well, however, if a $d_{x^{2}-y^{2}}$ wave component of the order parameter and faceting of the grain boundary are taken into consideration. Based on these considerations, it was expected that single grain boundaries will spontaneously generate magnetic flux $[5,6]$. We report evidence of the existence of this self-generated flux, obtained by direct imaging of single grain boundaries with scanning SQUID microscopy (SSM).

\section{EXPERIMENTAL TECHNIQUES}

The samples used for the experiments were high-quality, c-axis-oriented films of $\mathrm{YBa}_{2} \mathrm{Cu}_{3} \mathrm{O}_{7-x}$ grown by standard pulsed laser deposition on various bicrystalline $\mathrm{SrTiO}_{3}$ substrates to a thickness of 20$150 \mathrm{~nm}$. A grain boundary we investigated extensively is the asymmetric $45^{\circ}$ [001] tilt boundary, which is otherwise fabricated by biepitaxy. An image of the surface of such a sample, taken with tapping-mode atomic force microscopy (AFM), is presented in Fig. 1. This micrograph clearly reveals

(1) Present address: Dept. of Physics, Princeton University, Princeton, NJ 08536, U.S.A. 


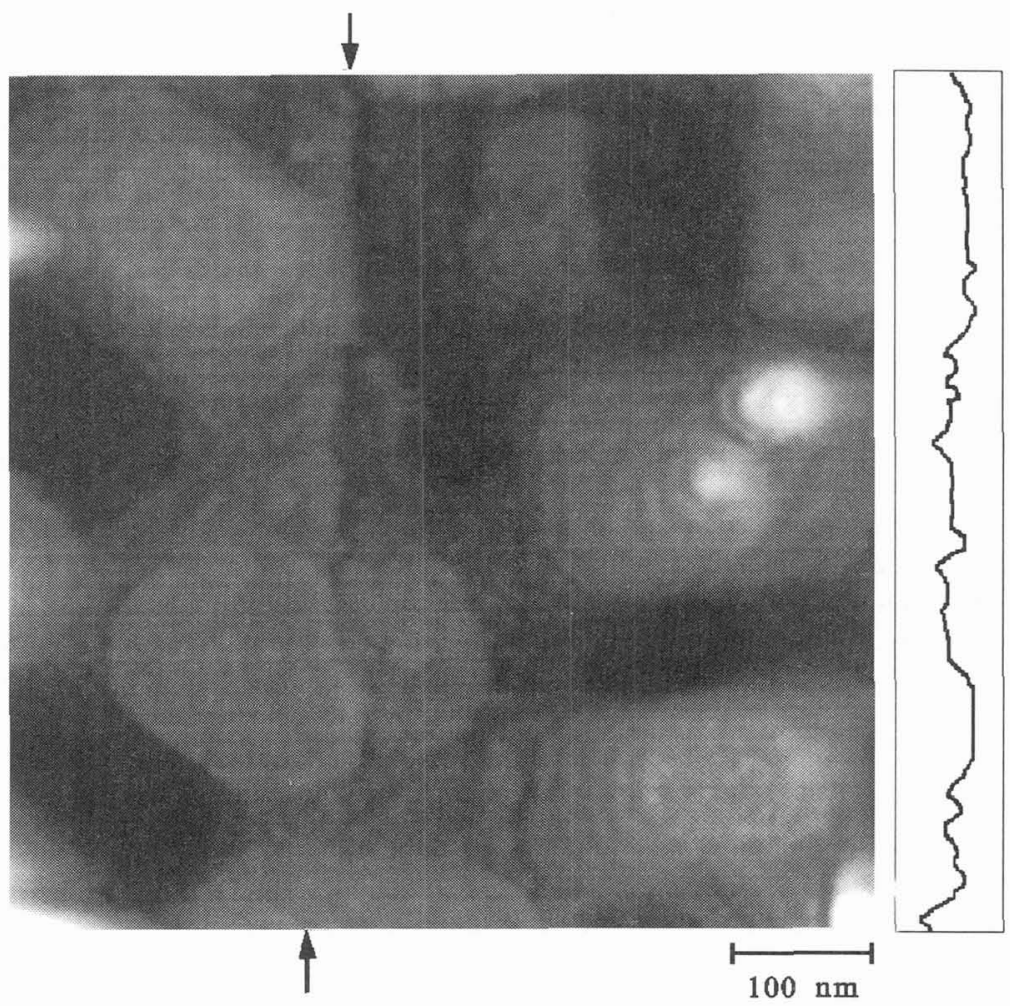

Figure 1: AFM image of the surface of an $\simeq 150$-nm-thick $\mathrm{YBa}_{2} \mathrm{Cu}_{3} \mathrm{O}_{7-x}$ film with an asymmetric $45^{\circ}$ [001] tilt boundary. The location of the grain boundary is indicated by arrows. The meandering path of the boundary has been replotted at the right of the figure. From Ref. [7].

the meandering of the grain boundary line. Typically, facets and continuously curved sections induced by the growth islands of the film are found on all length scales $<100 \mathrm{~nm}$, in agreement with published reports (see e.g. Ref. [8]).

\section{3. $I_{\mathrm{c}}\left(H_{\mathrm{a}}\right)$ CHARACTERISTICS}

\subsection{Experimental Results}

The asymmetric $45^{\circ}$ boundaries display an anomalous dependence of the critical current $I_{\mathrm{c}}$ on a magnetic field $H_{\mathrm{a}}$ applied in the boundary plane [5, 9-11]. An example of such an $I_{\mathrm{c}}\left(H_{\mathrm{a}}\right)$ curve is shown in Fig. 2. Except for a change of the absolute value of $I_{c}$, these characteristics do not change significantly as a function of temperature or oxygen doping [11]. One of their striking features is the small critical current in zero field $I_{\mathrm{c}}(0)$, which is significantly exceeded at some fields $H_{\mathrm{a}}^{*} \neq 0$. Such behavior is only consistent with standard junction theories [12] if self-field effects or trapped magnetic flux quanta are present. Both of these effects have to be ruled out in the present case [11]. 




Figure 2: Critical current as a function of applied magnetic field for an asymmetric $45^{\circ}$ [001] tilt boundary in an $\mathrm{YBa}_{2} \mathrm{Cu}_{3} \mathrm{O}_{7-x}$ film at $4.2 \mathrm{~K}$ (film thickness: $15 \mathrm{~nm}$, junction width: $16 \mu \mathrm{m}$, from Ref. [11]).

\subsection{Model}

The anomalous $I_{\mathrm{c}}\left(H_{\mathrm{a}}\right)$ characteristics can be remarkably well explained by a $d_{x^{2}-y^{2}}$ symmetry component of the superconducting order parameter (see e.g. Ref. [13]) and by the observed grain boundary faceting $[6,10,11]$. For example, in the simplest case, for a $d_{x^{2}-y^{2}}$ superconductor the density of the supercurrent $J(x)$ flowing across a facet is given by $J(x)=\prod_{m=1,2}\left(\left(\sin \alpha_{m}\right)^{2}-\left(\cos \alpha_{m}\right)^{2}\right) \sin \varphi(x)$ [14], where $\alpha_{m}(m=1,2)$ is the smallest angle between the facet plane and a principal axis of grain $m$, and $\varphi(x)$ is the difference of the phases of the order parameters. Concerning the critical current density $J_{c}(x)$, this implies that for some facet orientations $J_{c}\left(x_{\pi}, H_{\mathrm{a}}=0\right)<0$, so that the local current crosses the boundary in the opposite direction of $I_{\mathrm{c}}$ (see Fig. 3). These facets are called $\pi$ facets because for them the grain misorientation causes a shift of $\varphi(x)$ by $\pi$. If a field $H_{\mathrm{a}}$ is applied, a gradient $\nabla \varphi(x) \propto H_{\mathrm{a}}$ is induced. For some values $H_{\mathrm{a}}^{*}$ of $H_{\mathrm{a}}$ this leads to $\pi<\varphi\left(x_{\pi}\right)<2 \pi$, so that $J_{\mathrm{c}}\left(x_{\pi}, H_{\mathrm{a}}^{*}\right)>0$. Thus, $I_{\mathrm{c}}\left(H_{\mathrm{a}}^{*}\right)$ may be significantly larger than $I_{\mathrm{c}}(0)$. Exact calculations reproduce the measured $I_{\mathrm{c}}\left(H_{\mathrm{a}}\right)$ patterns well indeed.

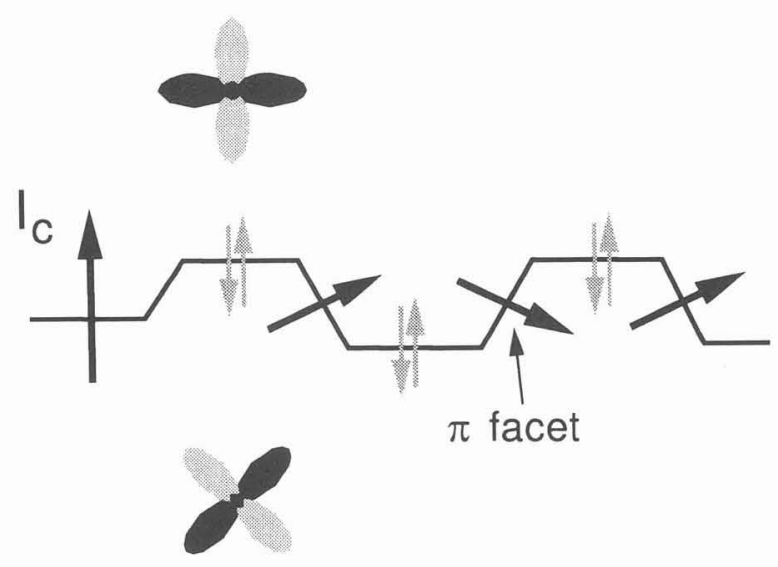

Figure 3: Sketch of the spatial distribution of $I_{c}$ flowing across a faceted boundary. The arrows indicate the direction of the current across the individual facets. 
For zero current bias, a highly interesting behavior has been predicted for the grain boundaries because the phase shift of the $\pi$ facets is expected to induce a. Josephson current. If these spontaneous currents exist, they will generate a disordered pattern of unquantized magnetic flux at the boundary $[5,6]$. To clarify whether magnetic flux is indeed generated by grain boundaries, we imaged the magnetic fields on the sample surface with high-resolution SSM [15, 16]. In the instrument used [16], a superconducting pickup loop, an integral part of a low- $T_{\mathrm{c}}$ SQUID, is scanned a few microns above the sample surface to image the local magnetic fields. Figure 4 shows an SSM image of a $1024 \times 256 \mu \mathrm{m}^{2}$ area of a $45^{\circ}$ grain boundary imaged with a $4-\mu \mathrm{m}$-diameter pickup loop. The sample was cooled in a field smaller than $1 \mathrm{mG}$. One bulk vortex pair is visible in the image, as well as spontaneously generated flux in the boundary. This flux is randomly distributed and also changes its sign randomly, with the observed fine-scale variations limited only by the spatial resolution $(\approx 4 \mu \mathrm{m})$ of the instrument. The characteristic features of this flux are found to be consistent in all respects with the expectations of the model based on the $d_{x^{2}-y^{2}}$ wave symmetry component of the superconductor and faceting [17].

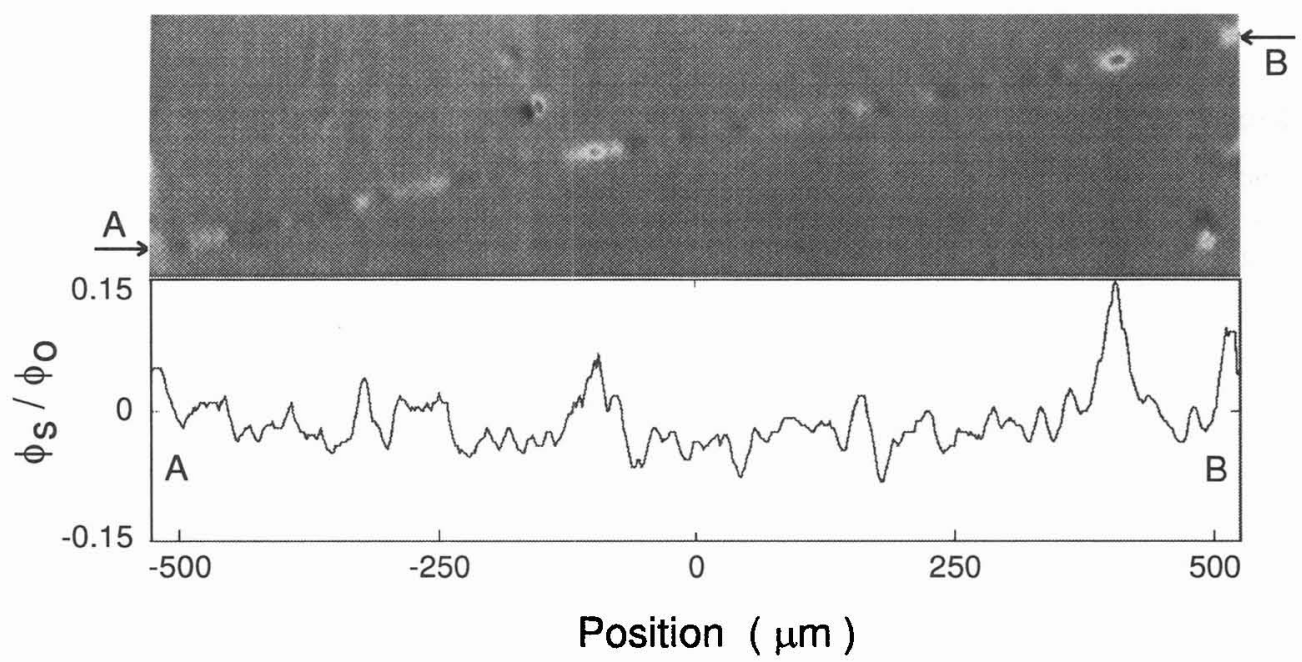

Figure 4: Scanning SQUID microscope image of a $1024 \times 256 \mu \mathrm{m}^{2}$ area including an asymmetric $45^{\circ}$ [001] tilt $\mathrm{YBa}_{2} \mathrm{Cu}_{3} \mathrm{O}_{7-x}$ bicrystal grain boundary. The image was taken at $4.2 \mathrm{~K}$ without an applied magnetic field after cooling the sample in a magnetically shielded environment $(<1 \mathrm{mG})$. The arrows indicate the location of the boundary. From Ref. [7].

\section{IMPLICATIONS OF A $d_{x^{2}-y^{2}}$ SYMMETRY COMPONENT ON GRAIN BOUNDARY PROPERTIES AND DEVICE APPLICATIONS}

The results described above have several significant implications, both for our understanding of the properties of grain boundaries in high- $T_{c}$ superconductors and for their applications. A more detailed discussion can be found in Refs. [6] and [17].

- Experimental proof has been presented for the presence of $\pi$ facets at grain boundaries in YBa2$\mathrm{Cu}_{3} \mathrm{O}_{7-x}$ films. The $\pi$ facets are the most prominent for asymmetric $45^{\circ}$ boundaries, independent of oxygen concentration and temperature. This provides further evidence of a $d_{x^{2}-y^{2}}$ symmetry component of the order parameter in $\mathrm{YBa}_{2} \mathrm{Cu}_{3} \mathrm{O}_{7-x}$. 
- Transport properties measured in practical grain boundary experiments reflect the averaged behavjor of inhomogeneous junctions. The dependence of $J_{c}$ on grain boundary misorientation, the $I(V)$ characteristic, and the $I_{\mathrm{c}} R_{n}$ product are not known for the individual facets. Thus, characteristics measured in standard experiments are no direct measure of intrinsic grain boundary properties.

- The effects discussed provide a missing link to explain the contradicting results of the symmetry experiments performed by Tsuei et al. [3] on tricrystal rings and by Chaudhari and Lin [2] on biepitaxial polygons. The averaging effect [18] and the presence of self-generated flux will suppress differences of $I_{c}$ for the various hexagon sites in the biepitaxial samples. If they are taken into account, the results of the polygon experiment become consistent with a $d_{x^{2}-y^{2}}$ wave order parameter $[17,18]$.

- $I_{\mathrm{c}}\left(H_{\mathrm{a}}\right)$ patterns measured across asymmetric $45^{\circ}$ boundaries are a straightforward way to investigate the presence of $\pi$ junctions and possibly also of higher order symmetry components in other high- $T_{\mathrm{c}}$ cuprates.

- The magnetic flux is a potential source of noise for grain boundary junctions and for SQUIDs. It can modify the current-phase relation of the junction and enhance critical current fluctuations. Asymmetric $45^{\circ}$ boundaries are the worst in this respect.

- The flux will cause a paramagnetic component of the grain boundary susceptibility.

- Of the three orders of magnitude drop of $J_{c}$ for an increase of the grain boundary angle from $0^{\circ}$ to $45^{\circ}$, about one order of magnitude may be attributed directly to a $d_{x^{2}-y^{2}}$ wave symmetry component and to faceting [6].

\section{Acknowledgments}

We thank J.G. Bednorz, R. Berger, M. Bushan, A. Ellis, D. Ertas, P. Guéret, M. Ketchen, A.J. Millis, K.A. Müller, and T.M. Rice for useful discussions and their valuable support. Part of this work was done in the framework of the ESPRIT project 8132 "WELITTD-HTS," and supported financially by Bundesamt für Bildung und Wissenschaft, Bern, Switzerland. The scanning SQUID microscope was partially supported by the Consortium for Superconducting Electronics (Adv. Res. Projects Agency Contract No. MDA972-90-C-0021). M.S. is grateful for financial support by the Swiss Nationalfonds (PROFIL fellowship).

\section{References}

[1] Chaudhari P. et al., Phys. Rev. Lett. 60 (1988) 1653.

[2] Chaudhari P. and Lin S.Y., Phys. Rev. Lett. 72 (1994) 1084.

[3] Tsuei C.C. et al., Phys. Rev. Lett. 73 (1994) 593.

[4] Miller J.H. et al., Phys. Rev. Lett. 74 (1995) 2347.

[5] Chew N.G. et al., Appl. Phys. Lett. 60 (1992) 1516.

[6] Hilgenkamp H. et al., Phys. Rev. B (1996) in press.

[7] Mannhart J. et al., Proc. 10th Anniversary HTS Workshop on Physics, Materials and Applications, Houston, TX, March 12-16, 1996 (World Scientific, Singapore, in press).

[8] Alarco J.A. et al., Ultramicroscopy 51 (1993) 239. 
[9] Ivanov Z.G. et al, Proc. Beijing Int'l Conf. on High-Temperature Superconductivity, Z. Gan et al. Eds. (World Scientific, Singapore, 1992), p. 722.

[10] Copetti C.A. et al., Physica C 253 (1995) 63.

[11] Mannhart J. et al., Z. Phys. B (1996) in press.

[12] Tinkham M., Introduction to Superconductivity, 2nd Ed. (McGraw Hill, New York, 1996).

[13] Müller K.A., Nature 377 (1995) 133.

[14] Sigrist M. and Rice T.M., J. Phys. Soc. Jpn. 61 (1992) 4283.

[15] Rogers F.P., "A Device for Experimental Observation of Flux Vortices Trapped in Superconducting Thin Films," Master's Thesis, MIT, Boston, 1983.

[16] Kirtley J.R. et al., Appl. Phys. Lett. 66 (1995) 1138.

[17] Mannhart J. et al., Phys. Rev. Lett. (1996) submitted.

[18] Millis A.J., Phys. Rev. B 49 (1994) 15408. 